\title{
REVUE CRITIQUE D'ETUDES SUR LE SOUTIEN SOCIAL ET SA RELATION AVEC LE BIEN-ETRE DE PERSONNES ATTEINTES DE DEMENCE
}

\author{
LOUISE LEVESQUE \\ et \\ SYLVIE COSSETTE \\ Université de Montréal
}

\section{RESUME}

Plusieurs personnes atteintes de maladies démentielles vivent à la maison, malgré les exigences de cette prise en charge. Le rôle d'aidant est assumé le plus souvent par un membre de la famille en particulier, lequel est plus susceptible de ressentir l'impact de cette prise en charge. Cet article présente une revue critique de 21 recherches sur le lien entre le soutien social informel et le bien-être de ces aidants. Les diverses dimensions du soutien étudiées dans ces recherches sont analysées ainsi que les limites méthodologiques. Une discussion des résultats concernant les relations entre le soutien et le bien-être complète l'analyse. Il ressort que les chercheurs évaluent le soutien d'une façon limitée et variée. La majorité d'entre eux n'ont pas recours à une définition du soutien qui s'appuie sur des postulats clairement spécifiés ou sur une théorie pertinente à ce construit. La faiblesse et la diversité de la conceptualisation du soutien se reflètent dans les instruments de mesure utilisés qui consistent, dans près de $50 \%$ des études, à ne formuler que quelques questions ad hoc sur le soutien. La disponibilité du soutien, sa durée, le conflit, et la réciprocité font l'objet de peu d'études, Le modèle de l'effet tampon du soutien est examiné dans une seule étude. Compte tenu de la petite taille des échantillons, tous accidentels, le controble des variables confondantes s'avère modeste. Lorsque les chercheurs utilisent une approche évaluative pour mesurer le soutien, les liens entre le soutien et le bienêtre sont plus souvent significatifs que lorsqu'ils recourent, à une approche descriptive. Dans les recherches futures, les chercheurs devront veiller à une meilleure articulation entre les notions théoriques proposées dans les recherches sur la prise en charge et celles issues des études sur le soutien social en général. En plus de renforcer les devis et d'entreprendre des recherches longitudinales, il apparaît essentiel de comprendre le mécanisme d'action du soutien vis-à-vis le bien-être de ces aidants. Dans cette perspective, une avenue consisterait à étudier la séquence des relations entre le stress, le soutien, les stratégies de coping, et le bien-être; d'autant plus qu'actuellement certains chercheurs intéressés à la prise en charge des personnes dèmentes s'appuient sur un cadre conceptuel du stress qui considère le soutien comme un facteur médiateur. Néanmoins, le travail effectué jusqu'à présent offre des pistes pour les études futures.

Nombreuses sont les personnes atteintes de maladies démentielles qui vivent dans leur milieu naturel. La plupart des familles désirent que leur parent malade 
demeure à la maison le plus longtemps possible, malgré les exigences et la complexité de cette prise en charge (Given, Collins, \& Given, 1988; Ory et al,, 1985). Cette prise en charge est le plus souvent assurée par un membre de la famille en particulier (Ory et al., 1985; Pruchno, 1990; Tennstedt, McKinlay, \& Sullivan, 1989). En effet, la disponibilité des membres de la famille à donner des soins suit ce que Shanas (1979) appelle le principe de substitution, c'est-à-dire qu'une seule personne assume principalement le rôle d'aidant. L'appellation saidant nature] principal», utilisée couramment dans les écrits, témoigne de cette réalité. Cette substitution s'effectue selon un ordre déterminé. Lorsqu'ils sont présents, les conjoints(es) constituent la première ligne d'aidants. En leur absence, ce sont les enfants adultes, particulièrement les filles et les belles-filles, qui prennent la relève, puis les parents plus éloignés, et finalement les amis et les voisins (Johnson \& Catalano, 1983). Actuellement, les chercheurs s'efforcent d'identifier les facteurs liès au bien-être des aidants principaux qui, à cause de leur rôle central, sont susceptibles de ressentir plus fortement que les autres membres de la famille, l'impact de la prise en charge. Le soutien social informel figure parmi ces facteurs. Ce choix s'explique par le fait que plusieurs recherches sur le soutien social en général (c'est-à-dire celles traitant spécifiquement du soutien auprès de différentes populations) indiquent une association entre le soutien social et différentes mesures de bien-être (Cohen \& Wills, 1985; Gottlieb \& Selby, 1989). De plus, l'étude de cette variable demeure importante puisque, de par sa nature, elle est en soi modifiable par des interventions appropriées.

Le but de cet article consiste à présenter une revue critique des recherches qui examinent les liens entre le soutien social informel et le bien-être de l'aidant principal d'une personne atteinte de démence. Les diverses dimensions du soutien mesurées dans les recherches relevées seront tout d'abord analysées; un examen méthodologique de ces études suivra ainsi qu'une discussion de leur résultats. L'article se terminera par la formulation de recommandations pour la recherche et la pratique clinique.

Les études furent relevées à partir de nos propres recensions d'écrits et celles effectuées par d'autres auteurs (Barer \& Johnson, 1990; Baumgarten, 1989; Dillehay \& Sandys, 1990; Gallo, 1990; Malonebeach \& Zarit, 1991; Montgomery, 1990; Morris, Morris, \& Britton, 1988). La période couverte dans cette recension s'étend de 1980 au début de 1991. Nous avons exclu de cette revue, les recherches portant uniquement sur la description du soutien puisque le thème privilégié dans cet article concerne la relation entre le soutien et le bien-être, tant psychologique, physique que social, et considéré comme une variable dépendante. Nous avons aussi exclu les recherches dont l'échantillon comprenait moins de $85 \%$ d'aidants de personnes atteintes de démence puisque notre intérêt porte sur la prise en charge d'un tel groupe de personnes. La démence est une maladie qui entrave profondément toutes les facettes du fonctionnement d'une personne et la diversité des troubles qu'elle entraîne nécessite des soins variés et particuliers de la part des aidants.

Au total, nous avons analysé 21 recherches portant sur 14 échantillons différents. La différence entre le nombre de recherches et celui des échantillons tient à deux situations. La première vient du fait que des parties de résultats obtenus auprès d'un même échantillon sont publiées dans des articles différents. La deuxième situation plus difficile à départager, se présente lorsque les auteurs 
rapportent des résultats préliminaires et des résultats finaux; dans ces cas, les résultats portent sur un échantillon dont la taille seulement est différente. Pour fin de clarté, nous avons décidé de considérer ces études comme différentes mais portant sur un "même» échantillon puisque les sujets sont recrutés et sélectionnées de la même façon.

\section{LES DIMENSIONS MESUREES DU SOUTIEN}

Avant de présenter l'analyse des dimensions du soutien mesurées dans les écrits relevés, une brève définition de ce construit s'impose. Si nous considérons l'expression «soutien social» d'une façon nominale, elle semble facile à définir à première vue. Tel n'est pas le cas puisqu'il existe plusieurs définitions de ce construit. En 1981, Barrera remarque que le soutien social n'est pas un construit conceptualisé et opérationnalisé de façon homogène. Bien que des progrès ont été réalisés quant à la conceptualisation de ce construit, il demeure qu'il n'existe pas encore une définition qui fasse l'unanimité des théoriciens du soutien social. Quoiqu'il en soit, suite à une recension d'écrits, Gottlieb et Selby (1989) dégagent une définition qui, à leur avis, tient largement compte de la conceptualisation de ce construit dans les recherches sur le soutien social en général. Selon cette définition:

le concept de soutien social informel fait référence à un processus d'interaction dans les relations entre des personnes. Ce processus favorise, notamment, le coping, l'estime de soi, le sens de lappartenance et la compétence au moyen d'échanges actuels ou prèvisibles de ressources matérielles ou psychologiques (p. 8, notre traduction).

Cette dèfinition met en lumière un fait important, soit que le soutien s'exprime au cours d'échanges interpersonnels, c'est-à-dire qu'il prend naissance lors d'un processus d'interaction. De plus, l'utilisation du terme "favorise» laisse entendre que le soutien s'avère plus souvent lié de façon bénéfique aux différents elèments énoncés dans la définition.

Pour analyser les dimensions du soutien mesurées dans les écrits relevés, nous avons élaboré une grille incluant 10 dimensions. Cette grille est basée en majeure partie sur celle utilisée par Stewart (1989) pour examiner les dimensions contenues dans les instruments du soutien social élaborés par des chercheurs en sciences infirmières. Stewart a construit sa grille à partir d'une revue des dimensions identifiées par des chercheurs clés dans le domaine du soutien social en général (Barrera, 1986; Cohen \& Syme, 1985; House \& Kahn, 1985; Shinn, Lehmann, \& Wong, 1984; Tardy, 1985). Notons que les dimensions relevées par Stewart s'apparentent à celles identifiées par Gottlieb et Selby (1989) lors de leur révision des perspectives d'évaluation du soutien social.

Le tableau 1 présente les 21 recherches par ordre chronologique et il fournit: (a) une description de l'échantillon, (b) un résumé des principales analyses utilisées pour examiner l'échantillon ou la robustesse de la mesure du soutien dans les cas oủ les auteurs les rapportent, (c) les analyses utilisées pour vérifier les hypothèses de relations entre le soutien et le bien-être, et (d) les dimensions du soutien mesurées. Les recherches dont le nom de l'auteur est précédé du sigle (Q@) portent sur un échantillon que l'on retrouve aussi dans une autre étude. Sauf dans deux recherches, tous les auteurs analysent les relations entre le soutien 
et le bien-être, en considérant séparément les différentes dimensions de ce construit telles qu'ils les ont retenues dans leur étude. Dans les deux cas d'exception (Haley, Bartolucci, Levine, \& Brown, 1987; Morris, Morris, \& Britton, 1989), les auteurs utilisent un score composé, c'est-à-dire un score qui rassemble les moyennes obtenues à diverses dimensions, pour tester la relation entre le soutien et le bien-être. De plus, il peut arriver que des auteurs regroupent des éléments d'une même dimension; dans ces cas, il s'agit d'un score global. Voyons maintenant les dimensions relevées dans les 21 recherches.

\section{La taille du réseau social}

Il s'agit du nombre de contacts avec les personnes faisant partie du réseau social ou de la fréquence des contacts avec ces personnes. La mesure de ces éléments permet d'obtenir un indice du réseau social (social network index) et d'évaluer l'intégration sociale. Tel que le mentionnent Gottlieb et Selby (1989), cette approche est surtout utilisée en épidémiologie où l'on examine les relations entre le degré d'intégration sociale et la santé. Il semble qu'un plus grand risque de mortalité survient parmi les sujets les plus isolés sur le plan social. Tel qu'illustré au tableau 1, dans neuf études, les auteurs mesurent cette dimension (Fiore, Becker, \& Coppel, 1983; Fiore, Coppel, Becker, \& Cox, 1986; Gilhooly, 1984; Gilleard, Belford, Gilleard, Whittick, \& Gledhill, 1984; Haley et al., 1987; Kielcolt-Glaser, Dyer, \& Shuttleworth, 1988; Morris et al., 1989; Niederehe \& Scott, 1987; Zarit, Reever, \& Bach-Peterson, 1980).

\section{La présence de confidents}

Selon Cohen et Wills (1985), la présence de confidents s'est avérée significativement liée au bien-être dans plusieurs recherches sur le soutien social en général. A leur avis, les liens s'expliquent par le fait que la présence de telles personnes implique en soi la disponibilité, notamment, d'un soutien offrant de l'information (informational support) ou favorisant l'estime de soi (esteem support). D'ailleurs, la présence d'un confident est souvent considérée comme une mesure d'intimité sociale (Gottlieb \& Selby, 1989). Seulement deux études considèrent cette dimension (Niederehe \& Scott, 1987; Pruchno, 1990).

\section{La source du soutien}

Cette dimension porte sur l'identification des membres du réseau avec lesquels une personne échange du soutien (e.g., la famille, les amis, etc.). Dans les recherches sur les aidants, les deux sources de soutien les plus considérées sont la famille seulement ou la famille et les amis. Dans ce dernier cas, il s'agit d'un score global qui ne permet pas de distinguer spécifiquement la source puisqu'il tient compte de la moyenne obtenue à ces deux sources (e.g., George \& Gwyther, 1986, tableau 1). Une telle distinction serait pertinente pour deux raisons. Premièrement, les types de soutien (voir no. 6) ainsi que l'intensité de l'échange varient, possiblement, selon la source. Par exemple, le soutien venant des amis peut avoir comme fonction de procurer des activités sociales ou récréatives tandis que la famille peut davantage procurer du soutien émotif ou du moins, en procurer d'une façon plus intense. Deuxièmement, Veiel (1985) souligne que la source du soutien peut en teinter son évaluation. Un soutien venant de la famille peut faire 
en sorte que les attentes d'une personne à l'endroit du soutien soient plus précises ou mieux connues; le soutien échangé serait alors évalué selon des attentes qui peuvent être différentes de celles où il provient d'amis ou de voisins.

Dans quelques recherches seulement, les auteurs ne font pas de distinction entre les sources du soutien informel et du soutien formel venant des professionnels (Pruchno, 1990; Quayhagen \& Quayhagen, 1988; Robinson, 1989; Zarit, Tood, \& Zarit, 1986). En effet, ils utilisent un score global en totalisant les scores obtenus à ces deux sources. Pruchno (1990) s'avère la seule à vérifier si le fait d'utiliser un score global fait varier le lien entre le soutien et le bien-être.

Les études où l'on ne distingue pas les sources de soutien ne peuvent guider adéquatement l'intervention clinique puisqu'elles ne précisent pas le réseau qu'il conviendrait de renforcer. Mentionnons que dans cinq recherches (Fiore et al., 1983, 1986; Kielcolt-Glaser et al., 1988; Pagel \& Becker, 1987; Pagel, Erdly, \& Becker, 1987), on demande aux aidants interviewés d'énumérer les personnes de leur réseau social sans toutefois spécifier le lien qu'ils ont avec ces personnes. Ainsi, la source du soutien n'est pas identifiée.

\section{La disposition (soutien reçu ou soutien disponible)}

Cette dimension se considère sous deux angles: le soutien effectivement échangé ou le soutien perçu comme pouvant être disponible. Dans ce dernier cas, il s'agit de la peception qu'une personne a de la disponibilité d'un soutien auquel elle pourrait recourir en situation de besoin. Selon Wethington et Kessler (1986), le soutien perçu comme disponible s'avère plus important que le soutien reçu, comme prédicteur d'adaptation à des situations de stress. Cette perception donnerait à une personne l'assurance psychologique qu'elle peut, au besoin, recourir a ce soutien. D'ailleurs, selon Gottlieb et Selby (1989), la perception de la disponibilité du soutien reflète «the psychological sense of support» (p. 13). Plus encore, Cohen et Wills (1985), suite à une revue colossale des recherches sur le soutien social, affirment que l'effet tampon du soutien sur le stress est le plus souvent mis en évidence lorsque les chercheurs mesurent la disponibilité. Dans les écrits, lorsqu'on parle d'effet tampon du soutien sur le stress, il est d'usage de se référer à l'hypothèse selon laquelle le soutien social, lors de situations de stress, protège les personnes des effets adverses du stress (une discussion sur cet effet tampon apparaît dans la conclusion).

Dans une seule recherche (Fiore et al., 1986), les auteurs tiennent compte de la disponibilité tandis que dans 10 recherches, on mesure l'aide reçue (Clipp \& George, 1990a, b; Gilhooly, 1984; Gilleard et al., 1984; Gilleard, Gilleard, Gledhill, \& Whittick, 1984; Niederehe \& Scott, 1987; Pruchno, 1990; Quayhagen \& Quayhagen, 1988; Robinson, 1989; Zarit et al., 1986).

\section{La durée des liens de soutien}

Cette dimension concerne la longévité du soutien. Une recherche (Clipp \& George, 1990b) porte sur cette dimension qui, par ailleurs, revêt une importance particulière dans la situation de prise en charge d'une personne atteinte de démence puisque cette maladie évolue sur une longue période et qu'il s'agit d'une situation de stress chronique. Les différents membres du réseau peuvent, avec le temps, ressentir de l'épuisement et diminuer leur participation dans l'échange de 


\section{TABLEAU 1}

\section{Aspects méthodologiques}

\begin{tabular}{|c|c|c|c|}
\hline Auteurs & $\begin{array}{l}\text { Description de } \\
\text { P'échantillon }\end{array}$ & Principales analyses & Dimensions du soutien \\
\hline Zarit et al. (1980) & $\begin{array}{l}n=29 \text { ( } 18 \text { conjoind[es], } \\
11 \text { filles) } \\
\text { cohabitation ps } \\
\text { rec: services } \\
\text { spécialisés }\end{array}$ & $\begin{array}{l}\text { H: } r \text { de Pearson en } \\
\text { considérant echantillon } \\
\text { total, conjoints(es) } \\
\text { et filles }\end{array}$ & $\begin{array}{l}\text { Fréquence mensuelle des visites au } \\
\text { parent malade des membres de la } \\
\text { famille autres que l'aidant prin- } \\
\text { cipal }\end{array}$ \\
\hline Fiore et al, (1983) & $\begin{array}{l}n=44 \text { conjoints(es); } \\
11 \text { conjoints(es) } \\
\text { malades en } \\
\text { hébergement } \\
\text { ree; diverses sources }\end{array}$ & $\begin{array}{l}\text { Pas de liens entre la } \\
\text { conforntité sociale et } \\
\text { mesures de soutien; et } \\
r \text { non sign, entre } \\
\text { «aidantos et } \\
\text { «dérangeanto selon les } \\
4 / 5 \text { types de soutien. } \\
\text { Pas de différence entre } \\
\text { gr en hébergement et à } \\
\text { domicile selon VT et } \\
\text { VD } \\
\text { H: régressions pas d̀ } \\
\text { pas (cinq types de sou- } \\
\text { tien dans les equations) }\end{array}$ & $\begin{array}{l}\text { Echelle des auteurs, basée sur l'ins- } \\
\text { trument de Hirsch (1980). Le } \\
\text { répondant énumère un maximum } \\
\text { de } 10 \text { personnes faisant partie de } \\
\text { son réseau social. Pour chaque } \\
\text { personne énumérée, il se prononce } \\
\text { sur la fréquence de contacts men- } \\
\text { suels et sur le degré selon lequel les } \\
\text { relations sont perçues uaidantess } \\
\text { et rdérangeantess et ce, pour } \\
\text { chacun des cinq types de soutien } \\
\text { présentés dans l'échelle }\end{array}$ \\
\hline Gilhooly (1984) & $\begin{array}{l}n=37 \text { ps } \\
\text { cohabitation }(n=20) \\
\text { ree: centre de jour }\end{array}$ & $\mathrm{H}: r$ de Pearson & $\begin{array}{l}\text { Fréquence mensuelle de contacts } \\
\text { avec la famille et avec les amis; } \\
\text { satisfaction de I'aide recue de la } \\
\text { parenté } \\
\text { Quantité d'ajde instrumentale ou } \\
\text { émotive recue de la famille, des } \\
\text { amis, et des voisins (SG) } \\
\text { Items tirés du OARS (Duke } \\
\text { University Center for the Studies } \\
\text { of Aging and Human Develop- } \\
\text { ment, 1978) }\end{array}$ \\
\hline $\begin{array}{l}\text { Gilleard, Belford, } \\
\text { Gilleard, Whittick, } \\
\text { et Gledhill (1984) }\end{array}$ & $\begin{array}{l}n=92 \text { (conjoints[es], } \\
\text { enfants-adultes, etc.) } \\
\text { cohabitation ps } \\
86 \% \text { de malades } \\
\text { déments } \\
\text { rec: centres de jour }\end{array}$ & $\begin{array}{l}\text { H: régressions pas à } \\
\text { pas }\end{array}$ & $\begin{array}{l}\text { Nombre hebdomadaire de visites } \\
\text { de la famille à l'aidant } \\
\text { Quantité d'aide reçue de la famille }\end{array}$ \\
\hline $\begin{array}{l}\text { (e) Gileard, Gil- } \\
\text { leard, Gledhill, } \\
\text { et Whittick } \\
\text { (1984) }\end{array}$ & $\begin{array}{l}\text { Même echantillon que } \\
\text { Gilleard, Bedford, } \\
\text { Gilleard, Whittick, et } \\
\text { Gledhill (1984), sauf } \\
n=45\end{array}$ & H: $r$ de Pearson & Quantité d'aide recue de la famille \\
\hline Scott et al. (1985) & $\begin{array}{l}n=23 \\
\text { conjoints[es], enfants) } \\
n=19 \text { membres de la } \\
\text { famille } \\
\text { cohabitation ps. } \\
\text { rec: diverses sources }\end{array}$ & $\begin{array}{l}\text { Analyse de contenu } \\
\text { des entrevues } \\
\text { H: comparaison des } \\
\text { moyennes entre les } \\
\text { groupes }\end{array}$ & $\begin{array}{l}\text { Entrevue non dirigé portant sur } \\
\text { les conflits au sujet de la prise en } \\
\text { charge, faite séparément avec } \\
\text { l'aidant et un membre de la } \\
\text { famille }\end{array}$ \\
\hline
\end{tabular}




\section{TABLEAU 1 (suite)}

Aspects méthodologiques

\begin{tabular}{|c|c|c|c|}
\hline Auteurs & $\begin{array}{l}\text { Description de } \\
\text { l'échantillon }\end{array}$ & $\begin{array}{l}\text { Principales } \\
\text { analyses }\end{array}$ & Dimensions du soutien \\
\hline $\begin{array}{l}\text { (28) Fiore et al. } \\
\text { (1986) }\end{array}$ & $\begin{array}{l}\text { Même échantillon } \\
\text { que Fiore et al. } \\
\text { (1983), sauf } n=68 \\
\text { conjoints(es); } \\
21 \text { conjoints(es) } \\
\text { malades en } \\
\text { hébergement }\end{array}$ & $\begin{array}{l}\text { Mêmes analyses que } \\
\text { Fiore et al. (1983) } \\
\text { pour conformité so- } \\
\text { ciale et différence } \\
\text { entre les deux gr.i } r \text { de } \\
\text { Pearson sign. entre } \\
\text { les diff, types du sou- } \\
\text { tien. } \\
\text { H: régression du type } \\
\text { hiérarchique. } \\
\text { A peu pres } \\
\text { pas de chevauchement } \\
\text { entre les items de sou- } \\
\text { tien et les trois VD }\end{array}$ & $\begin{array}{l}\text { Mëmes mesures que Fiore et al. } \\
\text { (1983), excepté que les répondants } \\
\text { se prononcent sur le degré de sa- } \\
\text { tisfaction/insatisfaction (qualité et } \\
\text { quantité) selon neuf types de sou- } \\
\text { tien, incluant la réciprocité (SG et } \\
\text { séparé). De plus, fréquence de } \\
\text { contacts (Fiore et al., 1983) et de } \\
\text { la disponibilité du soutien en re- } \\
\text { gard de chaque personne men- } \\
\text { tionnée dans la liste du réseau } \\
\text { social }\end{array}$ \\
\hline
\end{tabular}

\begin{tabular}{llll}
\hline George et & $n=510(54 \%$ con- & H: test de $t$ pour & Perception de plus de besoin de \\
Gwyther (1986) & joints[es]): & différence entre gr & soutien de la famille et des amis \\
& $34 \%$ parents malades & (besoin de plus de & (une question dichotomique oui/ \\
hébergés & soutien) et gr (pas de non, SG) \\
cohabitation: $53 \%$ & besoin) \\
& rec: diverses sources &
\end{tabular}

(4) Scott et al. Même échantillon que Même dêmarche que

Les répondants classés, selon: (a) (1986) Scott et al. (1985) Scott et al. (1985) sauf $\mathrm{n}=21$ plus de soutien qu'attendu, (b) suffisamment de soutien, et (c) pas assez. Soutien émotif ou instrumental venant de la famille (SG)

\begin{tabular}{|c|c|c|c|}
\hline $\begin{array}{l}\text { Zarit et al. } \\
\text { (1986) }\end{array}$ & $\begin{array}{l}\text { Au temps } 1, n=64 \\
\text { conjoints[es] } \\
\text { Au temps } 2 \text { ( } 2 \text { ans } \\
\text { d'intervalle), } n=45 \\
\text { conjoints(es): } 11 \text { con- } \\
\text { joints(es) en héberge- } \\
\text { ment }\end{array}$ & $\begin{array}{l}\text { H: régressions au } \\
\text { temps } 1 \text {; } \\
r \text { de Pearson au } \\
\text { temps } 2\end{array}$ & $\begin{array}{l}\text { Fréquence hebdomadaire d'aide } \\
\text { reçue des membres du réseau } \\
\text { informel et formel (SG) } \\
\text { Suffisance d'aide, une question: } \\
\text { ureçoit toute l'aide désirée (4)w a } \\
\text { ase sent dépassé et ne sait pas où } \\
\text { aller (1)» }\end{array}$ \\
\hline
\end{tabular}

rec: diverses 5ources

\begin{tabular}{lll}
\hline $\begin{array}{l}\text { Haley et al. } \\
\text { (1987) }\end{array}$ & $n=54$ (conjointes, 8; AF pour mesure du & Seore composé comprenant: no \\
conjoints, 7; enfants- soutien & d'amis; no de personnes intimes; \\
adultes, 30; autres, 9). H: régressions pas à no de contacts avec la famille et & nas \\
cohabitation ps & pamis; activités avec familles et \\
rec: diverses sources & amis; participation à des activités \\
& religieuses; satisfaction en regard \\
& des éléments mentionnés. Les iterns \\
& tirés surtout du Health and Daily \\
& Living Form (Moos, Cronkite, \\
& Billings, \& Finney, 1984)
\end{tabular}


TABLEAU 1 (suite)

Aspects méthodologiques

\begin{tabular}{|c|c|c|c|}
\hline Auteurs & $\begin{array}{l}\text { Description de } \\
\text { l'échantillon }\end{array}$ & $\begin{array}{l}\text { Principales } \\
\text { analyses }\end{array}$ & Dimensions du soutien \\
\hline $\begin{array}{l}\text { (4) Pagel et al. } \\
\text { (1987) }\end{array}$ & $\begin{array}{l}\text { Même echantillon } \\
\text { que Fiore et al. (1986), } \\
\text { sauf } n=68 \text { au temps } \\
1 \text { et } n=39 \text { au temps } \\
2 \text { (10 mois d'inter- } \\
\text { valle) }\end{array}$ & $\begin{array}{l}\text { AF et } r \text { de Pearson } \\
\text { pour mesure du } \\
\text { soutien. H: régressions } \\
\text { du type hiérarchique } \\
\text { temps } 1 \text {, temps } 2 \text {, et } \\
\text { temps } 1 \text { et } 2\end{array}$ & $\begin{array}{l}\text { Memes mesures que Fiore et al. } \\
\text { (1983), sauf que SG (moyenne des } \\
\text { scores obtenus aux cinq types de } \\
\text { soutien selon waides et wdérange- } \\
\text { ment») }\end{array}$ \\
\hline $\begin{array}{l}\text { (9) Pagel et } \\
\text { Becker (1987) }\end{array}$ & $\begin{array}{l}\text { Même échantillon que } \\
\text { Pagel et al. (1987) }\end{array}$ & $\begin{array}{l}\text { AF pour mesure du } \\
\text { soutien. H: régressions } \\
\text { du type hiérarchique }\end{array}$ & $\begin{array}{l}\text { Mesure de satisfaction/insatisfac- } \\
\text { tion (quantité, qualité) du soutien } \\
\text { recu de l'ensemble du réseau } \\
\text { social (selon chaque type de sou- } \\
\text { tien mais non selon chaque mem- } \\
\text { bre du réseau). Mesure semblable à } \\
\text { celle de Fiore et al. (1986) }\end{array}$ \\
\hline
\end{tabular}

\begin{tabular}{|c|c|c|c|}
\hline $\begin{array}{l}\text { Niederehe et } \\
\text { Scott (1987) }\end{array}$ & $\begin{array}{l}n=34 \text { ( } 17 \text { con- } \\
\text { joints[es] et } 17 \text { enfants- } \\
\text { adultes dont } 50 \% \\
\text { cohabitent avee le } \\
\text { parent malade) } \\
\text { rec: ps }\end{array}$ & $\begin{array}{l}\text { Alpha pour mesure } \\
\text { du soutien, } \\
\text { H: } r \text { de Pearson }\end{array}$ & $\begin{array}{l}\text { Echelle sur le partage des tâthes } \\
\text { (Scott, Wiegrand, \& Niederche, } \\
\text { 1984). Echelle de satisfaction du } \\
\text { soutien social des auteurs. Pré- } \\
\text { sence de confidents; taille du } \\
\text { réseau social; degré de s.suppor- } \\
\text { tivenesso }\end{array}$ \\
\hline $\begin{array}{l}\text { Kielcolt-Glaser } \\
\text { et al. (1988) }\end{array}$ & $\begin{array}{l}n=34 \text { ( } 20 \text { con- } \\
\text { joints[es]); } 10 \\
\text { parents en } \\
\text { hebergement; } \\
17 \text { cobabitent } \\
\text { rec: diverses sources }\end{array}$ & $\begin{array}{l}\text { H: régressions du } \\
\text { type hiérarchique }\end{array}$ & $\begin{array}{l}\text { Mêmes mesures que Fiore et al. } \\
\text { (1983) sauf SG pour kaides et } \\
\text { «dérangement» en considérant les } \\
\text { moyernes obtenues à chacun des } \\
\text { cinq types de soutien }\end{array}$ \\
\hline $\begin{array}{l}\text { Quayhagen et } \\
\text { Quayhagen (1988) }\end{array}$ & $\begin{array}{l}n=58 \text { ( } 43 \text { con- } \\
\text { joints(es]) } \\
\text { cobabitation ps } \\
\text { ree: services spécialisés }\end{array}$ & $\begin{array}{l}r \text { de Pearson et alpha } \\
\text { pour mesure du sou- } \\
\text { tien. H: corrélations } \\
\text { partielles en con- } \\
\text { trôlant pour le stress } \\
\text { lié à la condition du } \\
\text { parent malade }\end{array}$ & $\begin{array}{l}\text { Mesure du soutien informatif et } \\
\text { emotif reçus de la famille des } \\
\text { amis et des professionnels (SC } \\
\text { comprennant les deux types de } \\
\text { soutien et les trois sources) } \\
\text { Mesure inspirée de Schaefer, } \\
\text { Coyne, et Lazarus (1981) }\end{array}$ \\
\hline Robinson (1989) & $\begin{array}{l}n=78 \text { conjointes } \\
\text { ree: diverses sources }\end{array}$ & $\begin{array}{l}\text { AF et alpha pour } \\
\text { mesure de soutien } \\
\text { H: régressions du type } \\
\text { hiérarchique }\end{array}$ & $\begin{array}{l}\text { Echelle ISSB de Barrera, Sandler, } \\
\text { et Ramsay (1981) } \\
\text { Mesure de l'aide resue selon } \\
\text { quatre types de soutien en consi- } \\
\text { dérant la famille, les amis, les } \\
\text { voisins, et les professionnels (SG). } \\
\text { Echelle de l'auteur sur le désir de } \\
\text { plus d'aide concernant quatre } \\
\text { types de soutien }\end{array}$ \\
\hline
\end{tabular}


TABLEAU 1 (suite)

Aspects méthodologiques

\begin{tabular}{llll}
\hline & $\begin{array}{l}\text { Description de } \\
\text { Téchantillon }\end{array}$ & $\begin{array}{l}\text { Principales } \\
\text { analyses }\end{array}$ & Dimensions du soutien \\
\hline $\begin{array}{l}\text { Morris et al. } \\
(1989)\end{array}$ & $\begin{array}{l}n=20 \text { conjoints(es) } \\
\text { rec: diverses sources }\end{array}$ & H: $r$ de Pearson & $\begin{array}{l}\text { Echelle de Berkman (1983) sur } \\
\text { différents aspects du soutien: } \\
\text { réseau social, contacts sociaux, } \\
\text { disponibilité du soutien, personnes } \\
\text { les plus aidantes, etc. (5core com- } \\
\text { posé) }\end{array}$ \\
\hline
\end{tabular}

\begin{tabular}{|c|c|c|c|}
\hline $\begin{array}{l}\text { (8) Clipp et } \\
\text { George (1990a) }\end{array}$ & $\begin{array}{l}\text { Même échantillon que } \\
\text { George et } \\
\text { Gwyther (1986) }\end{array}$ & $\begin{array}{l}\text { H: régressions logis- } \\
\text { tiques }\end{array}$ & $\begin{array}{l}\text { Fréquence d'aide reçue de la } \\
\text { famille et des amis concernant } 12 \\
\text { tâches d'assistance (SG). } \\
\text { Perception de plus besoin d'aide } \\
\text { (George \& Gwyther, 1986) }\end{array}$ \\
\hline
\end{tabular}

\begin{tabular}{|c|c|c|c|}
\hline $\begin{array}{l}\text { (8) Clipp et } \\
\text { George (1990b) }\end{array}$ & $\begin{array}{l}\text { Même échantillon } \\
\text { que George et Gwyther } \\
\text { (1986), sauf } n=376 \\
\text { etude longitudinale } \\
1 \text { an d'intervalle }\end{array}$ & $\begin{array}{l}r=0,01 \text { entre les deux } \\
\text { mesures de soutien et } \\
\text { alpha } \\
\text { H: corrélations cano- } \\
\text { niques }\end{array}$ & $\begin{array}{l}\text { Stabilite ou changement du soutien. } \\
\text { Aide recue (Clipp \& George, } \\
\text { 1990a). } \\
\text { Perception de plus besoin d'aide } \\
\text { (George \& Gwyther, 1986) }\end{array}$ \\
\hline Pruchno (1990) & $\begin{array}{l}n=315 \text { conjoints(es) } \\
\text { rec: diverses sources }\end{array}$ & $\begin{array}{l}\text { Analyses de variance } \\
\text { indiquant aucune dif- } \\
\text { férence selon les trois } \\
\text { sources d'aide en } \\
\text { regard des VD; } \\
\text { H: test } t \text { sur } \\
\text { moyennes aux VD } \\
\text { selon aide recue ou } \\
\text { non }\end{array}$ & $\begin{array}{l}\text { Quantité d'aide reçue de la famille, } \\
\text { des amis, et des professionnels en } \\
\text { considérant } 15 \text { activités journil- } \\
\text { lières (SG). } \\
\text { Présence d'un confident }\end{array}$ \\
\hline
\end{tabular}

ps (pas spécifié); reo (recrutement); gr (groupe); H (tests pour vèrifier les hypothèses de liens entre soutien et bien-être); VI et VD (variables indépendantes et dépendantes); SG (score global, e' est-à-dire les différents types ou différentes sources calculés dans un même score); AF (analyses factorielles); alpha (alpha de Cronbach): (a) (étude portant sur un échantillon déjủ décrit dans une autre étude). Tous les échantillons sont accidentels et comprennent des aidants principaux dont le parent malade est à domicile, sauf si mentionné en hêbergement.

soutien. Notons que dans cette recherche, les variables de bien-être sont considérées comme prédicteurs de la durée et non comme variables dépendantes.

\section{Les types de soutien}

Le soutien peut prendre plusieurs formes et elles sont classifiées selon différents types ou fonctions, c'est-à-dire selon à quoi elles servent. Dans les recherches sur le soutien social en général, il existe plusieurs typologies (e.g., soutien émotif, instrumental, favorisant l'information, la socialisation, l'estime de soi, etc.) mais, aucune typologie n'obtient véritablement l'unanimité des chercheurs (Ryan \& Austin, 1989; Tilden, 1985). Actuellement, le seul consensus parmi les chercheurs concerne la reconnaissance de deux types de soutien, soit le soutien émotif et instrumental. Le soutien émotif comprend les échanges de soutien 
dont le but est de modifier des paramètres intrapsychiques tels que l'humeur, les attitudes, ou des processus cognitifs. Le soutien instrumental a pour but d'aider une personne à agir (Veiel, 1985). Pour leur part, Cohen et Wills (1985) recommandent de tenir compte des soutiens favorisant l'information et l'estime de soi, croyant que ces deux types de soutien procurent des ressources pour un large éventail de situations de stress.

Dans les recherches relevées, trois scénarios se dégagent: (a) six études ne spécifient pas les types de soutien (Gilleard, Belford, Gilleard, Whittck, \& Gledhill, 1984; Gilleard, Gilleard, Gledhill, \& Whittck, 1984; George \& Gwyther, 1986; Haley et al., 1987; Morris et al., 1989; Zarit et al., 1986); (b) le soutien émotif et instrumental (score global) sont mesurés dans deux études (Gilhooly, 1984; Scott, Roberto, Hutton, \& Slack, 1986), tandis que seulement le soutien instrumental est examiné, et de façon détaillée, dans quatre recherches (Clipp \& George, 1990a, b; Niederehe \& Scott, 1987; Pruchno, 1990); et (c) six études tiennent compte de plusieurs types de soutien (Fiore et al., 1983; Kielcolt-Glaser et al., 1988; Pagel \& Becker, 1987; Pagel et al., 1987; Robinson, 1989), mais seulement Fiore et al. $(1983,1986)$ les analysent séparément. Si nous tenons compte de la recommandation de Cohen et Wills (1985) à l'effet de considérer le soutien favorisant l'information et l'estime de soi, nous observons que le soutien favorisant l'information figure dans ces six recherches, tandis que celui favorisant l'estime de soi n'apparaît que dans une étude (Fiore et al., 1986).

Actuellement, les chercheurs reconnaissent l'importance de distinguer précisément les types de soutien pour pouvoir mieux identifier les besoins de soutien selon l'événement stressant. En d'autres termes, pour aider à l'avancement des connaissances sur le soutien, il conviendrait de connaître quels types précis de soutien sont générés selon la nature des événements stressants et selon qu'il s'agit d'une situation de crise ou non. Cohen et Wills (1985) ainsi que House et Kahn (1985) croient que la relation entre le soutien et le bien-être varie en fonction des types de soutien et des situations. La distinction des types de soutien est également importante lorsqu'il s'agit de tester l'effet tampon du soutien sur le stress. Cohen et Wills (1985) rapportent que cet effet tampon s'observe lorsque les ressources répondent d'une façon pertinente aux besoins engendrés par les événements stressants. Il va de soi que la connaissance précise des types de soutien dont une personne a besoin s'avère indispensable à l'implantation d'interventions appropriées.

Les prochaines dimensions que nous allons examiner portent sur la qualité de la relation de soutien. Pour mesurer la qualité, plutôt que de recourir à une approche descriptive comme dans le cas des dimensions énumérées précédemment, on utilise une approche évaluative. On demande alors à la personne de porter un jugement évaluatif sur le soutien, contrairement à l'approche descriptive oủ, par exemple, un répondant doit rapporter la fréquence de l'aide reçue, c'est-à-dire décrire un état de fait. Parmi les recherches relevées, nous en retrouvons un plus grand nombre où les deux types d'approches sont utilisées plutôt que l'une ou l'autre.

\section{Les aspects aidants (helpful) et dérangeants (upsetting) du soutien}

Selon Cohen et Wills (1985) et Gottlieb et Selby (1989), les théoriciens du soutien social s'accordent maintenant à dire que le soutien peut être non seule- 
ment une source d'aide, mais aussi une source de stress associée à des aspects dérangeants du soutien (envahissement de l'intimité, etc.). Tilden et Galyen (1987) qualifient cet aspect de «dark side of the social support». Il importe donc de distinguer les échanges de soutien aidant de ceux de soutien dérangeant. Telles qu'illustrées au tableau 1, ces deux dimensions sont mesurées dans trois recherches (Fiore et al., 1983; Kielcolt-Glaser et al., 1988; Pagel et al., 1987). Par ailleurs, Morris et al. (1989) ne considèrent que l'aspect aidant, et ce, dans un score composé. Pour leur part, Niederehe et Scott (1987) mesurent le «network supportiveness", sans toutefois définir cette expression ou sa mesure. Nous avons tout de même considéré cette mesure comme évaluant l'aspect aidant du soutien.

Notons que certains auteurs sur le soutien social en général examinent un aspect particulier du concept de "upsetting", à savoir le conflit (Rook, 1984; Tilden \& Galyen, 1987). Seulement une recherche (Scott, Roberto, \& Hutton, 1985) porte sur ce point bien que cliniquement, certains conflits sont observés dans les familles quant aux stratégies de la prise en charge (Zarit, Orr, \& Zarit, 1985).

\section{La satisfaction/insatisfaction}

Toujours dans le courant de pensée que le soutien ne peut être qu'aidant, des chercheurs choisissent d'examiner le degré de satisfaction/insatisfaction. Au tableau 1, nous retrouvons les études où des chercheurs utilisent cette mesure évaluative (Fiore et al., 1986; Gilhooly, 1984; Niederehe \& Scott, 1987; Pagel \& Becker, 1987). Pour leur part, Haley et al. (1987) considèrent la satisfaction dans leur score composé du soutien.

\section{La réciprocité dans l'échange de soutien}

Une même personne peut tantôt recevoir du soutien, tout comme elle peut en donner à d'autres moments. La réciprocité devient asymétrique si une personne reçoit plus de soutien qu'elle n'en donne ou la situation inverse. Lorsqu'une personne croit ne pouvoir rendre le soutien reçu de son entourage, elle peut décider de ne pas y recourir afin de préserver son estime d'elle-même ou son autonomie (Sandler \& Barrera, 1984; Tilden \& Stewart, 1985; Wentkowski, 1981). Stewart (1989) note que la réciprocité s'est avérée une dimension pertinente à scruter quant à la promotion de la santé. Selon Ward (1985), la réciprocité est un élément clé dans l'évaluation du soutien chez les personnes âgées qui souvent reçoivent plus de soutien qu'elles ne peuvent en donner. Cette asymétrie concourt à renforcer leur sentiment de dépendance et ainsi, leur bien-être peut être entaché. Il est raisonnable de penser qu'un échange asymétrique de soutien puisse exister dans la situation de prise en charge, mais seulement Fiore et al. (1986) examinent cette dimension.

\section{La suffisance du soutien}

Dans une recherche (Robinson, 1989), on demande aux aidants de se prononcer sur leur désir de recevoir plus d'aide en considérant quatre types de soutien. Par ailleurs, nous observons que dans les recherches de George et Gwyther (1986) et de Clipp et George (1990a, b) portant sur un même échantillon, on recourt à une question sans considérer les types de soutien pour mesurer cette 
dimension (tableau 1). La même stratégie est utilisée dans l'étude de Scott et al. (1986) et dans celle de Zarit et al. (1986).

En somme, dans la majorité des études, les auteurs évaluent le soutien d'une façon limitée en ne considérant que quelques dimensions. La disponibilité, la durée, le conflit, et la réciprocité s'avẻrent peu examinés. En plus d'être limitées, les dimensions étudiées varient d'une étude à l'autre. Nous ne pouvons identifier une même dimension qui aurait été retenue dans toutes les études. Une telle situation s'explique, en partie du moins, par le fait que la majorité des chercheurs ne recourent pas à une définition proposée par des théoriciens du soutien social pour choisir les dimensions scrutées. Certes, les chercheurs ne peuvent étudier toutes les dimensions souhaitées du soutien lorsque d'autres facteurs liés au bien-être sont aussi examinés et que la taille de l'échantillon est petite. Mais, dans un tel contexte, le recours à une définition du soutien qui s'appuie sur des postulats ou sur une théorie pertinente à ce construit (e.g., la théorie de l'échange social) aiderait à choisir les dimensions les plus critiques à retenir. Nous observons une telle tentative dans les études du groupe Fiore-Pagel (Fiore et al., 1983, 1986; Pagel \& Becker, 1987; Pagel et al., 1987) qui portent sur un même échantillon ainsi que dans celle de Kielcolt-Glaser et al. (1988). Ces auteurs s'appuient sur les travaux de Hirsch (1980) sur le soutien pour choisir les dimensions qu'ils étudient. Nous allons maintenant aborder l'examen de certains aspects méthodologiques en ce qui a trait aux recherches relevées.

\section{ASPECTS METHODOLOGIQUES}

\section{Les échantillons}

Tous les échantillons sont accidentels et les participantes ne sont pas recrutées à partir de la population. Le recrutement se fait parmi les aidants qui reçoivent des services spécialisés pour leur venir en aide (e.g., groupes de soutien, etc.), ou parmi ceux qui ont leur parent malade inscrit à un centre de jour, sur la liste d'un médecin ou d'un service de santé; dans certaines études, il y a des participantes recrutées par voies médiatiques (tableau 1). Ces échantillons sont donc peu représentatifs d'une population d'aidants. Les aidants qui font appel à des services particuliers peuvent être plus perturbés que d'autres par la prise en charge. De plus, dans les études transversales, et la grande majorité des recherches relevées le sont, on ne rejoint pas les participantes qui ont abandonné leur rôle d'aidant, et ce, dans une situation où les parents malades sont à un stade comparable d'évolution de la maladie. Le risque existe de retrouver dans les échantillons, des aidants que l'on peut qualifier de survivants ou de persévérants. Ce biais se retrouve aussi dans les recherches longitudinales, mais il demeure que dans de telles études, nous sommes plus en mesure de pouvoir connaître et comparer les facteurs liés au fait d'abandonner le rôle d'aidant. En somme, compte tenu des contraites de recrutement, la validité externe des études est limitée.

Huit des 14 échantillons sont formés de participants hétérogènes, par exemple, de conjoints(es), d'enfants adultes, etc. Egalement, les aidants ne cohabitent pas toujours avec le parent malade et, même plus, dans quatre échantillons, nous retrouvons aussi des aidants dont le parent malade est hébergé dans un établissement de santé. On n'examine pas toujours les effets de ces variables sur les résultats obtenus (tableau 1). Or, il est bien reconnu que le lien avec le parent 
malade et le fait de cohabiter ou non constituent des variables qui peuvent créer des artefacts s'ils ne sont pas contrôlés.

\section{Les devis}

Puisque seulement trois recherches sont longitudinales (Clipp \& George, 1990b; Pagel et al., 1987; Zarit et al., 1986), nous sommes peu renseignés sur la direction de la relation entre le soutien et le bien-être. Par exemple, est-ce qu'un soutien satisfaisant fait que les aidants sont moins déprimés ou est-ce que les aidants moins déprimés reçoivent plus de soutien?

\section{Les mesures du soutien}

Les façons de mesurer le soutien peuvent se regrouper selon quatre scénarios. Le premier comprend deux études (Quayhagen \& Quayhagen, 1988; Robinson, 1989) où les chercheurs utilisent un instrument propre au soutien et pour lequel certaines propriétés psychométriques ont été examinées, selon Heitzmann et Kaplan (1988) dans une revue des écrits sur les mesures du soutien social. Dans le deuxième scénario, il s'agit de situations où on recourt à un instrument utilisé dans des recherches sur le soutien social mais pour lequel, à notre connaissance, les qualités psychométriques ne sont pas rapportées. Dans cette catégorie, nous retrouvons la recherche de Morris et al. (1989) ainsi que cinq études où les auteurs utilisent le même instrument (Fiore et al., 1983, 1986; Kielcolt-Glaser et al., 1988; Pagel \& Becker, 1987; Pagel et al., 1987). Le troisième scénario comprend les deux recherches où on se sert d'items contenus dans des instruments qui ne sont pas propres au soutien (Gilhooly, 1984; Haley et al., 1987). Quant au quatrième scénario, il englobe toutes les autres études (près de $50 \%$ ) qui ont en commun le fait que les auteurs développent leur propre mesure qui se résume la plupart du temps à la formulation de quelques questions ad hoc, c'est-à-dire qui ne servent qu'à l'étude en cours.

Dans près de $40 \%$ des recherches, les instruments utilisés sont soumis à certaines analyses, comme par exemple, des analyses d'homogénéité, de corrélations entre les mesures du soutien utilisées ou des analyses factorielles jugées satisfaisantes par les auteurs. Dans deux études portant sur le même échantillon, les auteurs (Fiore et al., 1983, 1986) montrent que les scores du soutien social ne sont pas influencés par la conformité sociale (tableau 1, pour le détail de ces différentes analyses).

Tel que mentionné précédemment, dans deux études, les auteurs regroupent des dimensions diffèrentes du soutien dans un même score composé (Haley et al., 1987; Morris et al, 1989). De telles mesures ne permettent pas d'isoler la contribution spécifique de chaque dimension et renseignent peu quant à la compréhension de la relation entre le soutien et le bien-être. De plus, à l'intérieur de la dimension "types de soutien», Fiore et al. (1986) rapportent des coefficients de corrélation significatifs $(35 / 36)$ entre les neuf types de soutien examinés, indiquant ainsi, soit l'existence d'un certain chevauchement conceptuel ou encore, la possibilité que l'entourage ne se limite pas à offrir un seul type de soutien. 


\section{Les mesures de bien-être}

En consultant le tableau 2, nous constatons que pour l'ensemble des recherches, 10 facettes du bien-être sont mesurées. Dans la majorité des études, ces mesures portent sur des aspects "négatifs» comme la dépression, le fardeau, la tension, ou des symptômes psychiatriques contenus dans plusieurs mesures de santé mentale, etc. Cinq études seulement font exception en mesurant un ou des aspects positifs de santé mentale (George \& Gwyther, 1986; Gilhooly, 1984; Pruchno, 1990) ou la satisfaction de vie (George \& Gwyther, 1986; Haley et al., 1987), ou la satisfaction quant aux activités sociales (George \& Gwyther, 1986). De plus, Quayhagen et Quayhagen (1988) incluent la satisfaction de vie dans leur score composé de bien-être. Nous retrouvons cette tendance à considérer des aspects négatifs dans les recherches sur le soutien en général (Cohen \& Wills, 1985). Cependant, nous pouvons nous demander si la nature ou l'ampleur des liens entre le soutien et le bien-être changent selon que ce dernier construit est mesuré sous un angle positif ou négatif. Dans les études citées précédemment, les résultats apparaissent équivoques. Dans les neuf cas où on vérifie le lien entre le soutien et le bien-être mesuré positivement, il y en a cinq qui présentent une relation significative et positive entre ces deux construits.

Quant aux instruments, nous constatons qu'ils sont variés. Pour les deux facette du bien-être les plus retenues, soit la dépression et la santé mentale, la majorité des chercheurs recourent à une mesure dont les qualités psychométriques sont reconnues. L'échelle de dépression de Beck et Beamsderfer (1974) demeure la plus utilisée; parmi les instruments de santé mentale, nous retrouvons, par exemple, le SCL-90 de Derogatis, Rickels, et Rock (1976) et le Goldberg Health Questionnaire (Goldberg, 1978). En ce qui a trait aux autres mesures de bien-être, il s'avère que certaines d'entre elles sont élaborées par les auteurs dont certains rapportent quelques qualités psychométriques. La seule mesure composée de bien-être se retrouve dans l'étude de Quayhagen et Quayhagen (1988), mesure qui porte sur la moyenne obtenue à la satisfaction de vie, à la perception de la santé physique et mentale. Mis à part Fiore et al. (1986) qui, après un examen nominal des items, ne retrouvent pas de recoupement entre les items de mesure du soutien et ceux du bien-être, aucun autre auteur ne s'interroge sur cette possibilité. Pourtant, un recoupement entre ces mesures donneraient lieu à des liens artificiels entre le soutien et le bien-être.

\section{Les analyses statistiques}

La petite taille des échantillons constitue une limite dans le choix des tests statistiques servant à mesurer les liens entre le soutien et le bien-être. Dans une revue des écrits sur la santé des aidants naturels de personnes démentes, Baumgarten (1989) en s'appuyant sur Cohen (1977), considère un nombre de 84 participantes comme étant critique pour déceler d'une façon fiable, un coefficient de corrélation significatif d'une ampleur modérée ( $r$ de Pearson de 0,30 , $p \leq 0,05)$. Dans cinq études seulement, les échantillons ont plus de 84 participantes (Clipp \& George, 1990a, b; George \& Gwyther, 1986; Gilleard, Belford, Gilleard, Whittck, \& Gledhill, 1984; Pruchno, 1990). Compte tenu de la petite taille des échantillons, des analyses de corrélations sont souvent effectuées. Il est permis de penser que certaines associations pourraient être plus faibles en tenant compte de la multicollinarité entre les facteurs retenus. Dans les analyses de 
régression, il arrive le plus souvent que peu de variables autres que le soutien soient retenues dans l'équation. Dans l'étude de George et Gwyther (1986), les auteurs effectuent des différences de moyenne entre le groupe d'aidants rapportant avoir plus de besoin de soutien et celui de ceux qui n'éprouvent pas un tel besoin. Pour leur part, Scott et al. $(1985,1986)$, dans leurs deux études portant sur le même échantillon, ne comparent que nominalement les moyennes et n'effectuent pas de tests statistiques. Toujours à cause de la petite taille de la majorité des échantillons, le contrôle des variables confondantes s'avère très modeste. Dans le tableau 1, nous indiquons les analyses statistiques utilisées pour vérifier l'hypothèse de liens entre le soutien et le bien-être.

En somme, la faiblesse et la diversité de la conceptualisation du soutien se reflettent dans la grande majorité des mesures utilisées. Des échantillons accidentels souvent hétérogènes, le peu de contrôle de variables confondantes constituent des limites affectant la validité interne et externe des études. Ces limites peuvent certainement contribuer à expliquer les résultats équivoques. Malgré ces considérations, nous les analyserons pour tenter de dégager des tendances des études relevées.

\section{LA RELATION ENTRE LE SOUTIEN ET LE BIEN-ETRE}

Dans cette partie, nous n'examinons que les principaux résultats. Ceux concernant la disponibilité, le conflit et la réciprocité ne font pas l'objet de commentaires quant aux relations puisqu'ils sont étudiés par peu d'auteurs. Les résultats ne peuvent être examinés selon la source et les types de soutien; dans la majorité des recherches considérant ces deux dimensions, on ne distingue pas la source ou les types, mais plutôt, on utilise un score global pour chacune d'entre elles. De plus, nous excluons de notre analyse les résultats obtenus aux deux études utilisant un score composé du soutien car, à notre avis, un tel score demeure ambigu. Finalement, l'étude sur la durée (Clipp \& George, 1990b) ne figure pas dans le tableau 2 puisque le soutien est considéré comme une variable dépendante.

Le tableau 2 indique les relations étudiées entre les dimensions du soutien et celles du bien-être. Puisque chaque étude évalue plus d'un lien, la discussion porte sur ce point et non sur les études. En comparant les mesures descriptives et évaluatives du soutien, nous retrouvons moins de liens significatifs $(11 / 39)^{1}$ entre le soutien et le bien-être dans le cas des mesures descriptives. En effet, dans les études évaluatives, il y a 21/36 liens significatifs. En ce qui a trait aux mesures descriptives, il ressort que:

- Parmi les 13 situations où l'on mesure des aspects de la taille du réseau, nous observons quatre liens significatifs. Cette dimension du soutien semble donc peu reliée au bien-être quels que soient les aspects retenus.

- En ce qui a trait à la présence de confidents, dans l'étude de Niederehe et Scott (1987), il y a trois liens significatifs sur cinq tandis que dans celle de Pruchno (1990), nous n'en observons aucun. Notons que l'étude de Pruchno comporte 315 participantes et celle de Niederehe et Scott en comprend 34. Comme nous pouvons le constater les résultats ne sont pas constants.

- Parmi les nombreuses relations concernant la disposition mesurée sous l'angle de l'aide reçue, seulement deux sont significatives et elles sont associées 
différemment au bien-être. Dans l'étude de Quayhagen et Quayhagen (1988), plus les aidants reçoivent du soutien, plus ils rapportent un degré de bien-être élevé. Par contre, selon l'étude de Clipp et George (1990a), plus ces derniers reçoivent de l'aide, plus ils risquent de consommer des psychotropes. Cependant quoique significative, la valeur du coefficient exponentiel est faible $(1,02)$. Les auteurs attribuent cette faible valeur à l'étendue passablement large du score de la variable de soutien. Cette observation s'avère importante puisque, selon les résultats rapportés dans l'article de Clipp et George (1990b), chez les aidants du groupe qui reçoit peu de soutien durant un an, cette tendance ressort encore moins. Nous sommes en droit de penser que ces résultats équivoques peuvent être attribuables soit au type d'analyse utilisée (régression logistique) en fonction de la mesure des variables indépendantes retenues ou à la différence des échantillons dans ces deux études due à la perte de sujets dans la recherche longitudinal.

Par ailleurs, l'examen des résultats obtenus aux mesures évaluatives indique que:

- Dans quatre études (Fiore et al., 1983; Kielcolt-Glaser et al., 1988; Niederehe \& Scott, 1987; Pagel et al., 1987), il n'y a pas de lien significatif entre le soutien aidant (helpful) et le bien-être. Par contre, les liens entre le soutien dérangeant (upsetting) et le bien-être s'avèrent toujours significatifs dans les trois études où on le mesure (Fiore et al., 1983; Kielcolt-Glaser et al., 1988; Pagel et al., 1987). Dans leur étude longitudinale, Pagel et al. (1987) observent, au temps 1 comme au temps 2 , que seulement le soutien dérangeant explique la variation de la dépression, ce qui n'est pas le cas pour le soutien aidant. De plus, les changements dans le soutien dérangeant prédisent les changements dans la dépression entre le temps 1 et 2 . Les analyses factorielles effectuées par ces auteurs indiquent que le soutien aidant et le soutien dérangeant représentent deux construits distincts.

Dans ces trois études, on utilise le même instrument de mesure du soutien qui porte sur cinq types différents. Seuls Fiore et al. (1983) les considèrent dans l'analyse de régression, les autres auteurs ayant fait une moyenne à partir des scores obtenus à chacun des types. Vu sous l'angle du dérangement, deux des cinq types de soutien expliquent significativement $34,1 \%$ de la variance de la dépression. Il s'agit du soutien favorisant l'information $(28,8 \%)$ et l'aide instrumentale $(5,3 \%)$. Le premier résultat renforce la recommandation de Cohen et Wills (1985) à l'effet de tenir compte du soutien favorisant l'information. Par ailleurs, aucun type de soutien mesuré sous l'angle de l'aide n'est relié à la dépression. Enfin, notons que selon ces trois études, lorsque le soutien est dérangeant, le degré de dépression s'élève.

- La majorité des liens entre la satisfaction/insatisfaction du soutien et le bienêtre sont significatifs. Ces résultats s'apparentent à ceux d'autres études sur le soutien social en général, y incluent des échantillons de personnes âgées, où un bas degré de satisfaction en regard du soutien est lié à un degré élevé de dépression (Barrera, 1981; Hirsch, 1980; Krause, Liang, \& Yatomi, 1989).

La recherche de Pagel et Becker (1987) mérite d'être soulignée puisqu'elle examine l'effet tampon du soutien sur une variable portant sur la situation de prise en charge. L'étude indique, d'une part, que plus la satisfaction quant au soutien 
est élevée, moins les aidants éprouvent de la dépression. D'autre part, les auteurs analysent l'interaction entre la satisfaction du soutien et le degré de contrôle que les aidants croient avoir sur certaines situations de prise en charge (e.g., comportements imprévisibles du parent) ainsi que sur le degré selon lequel ils se blâment quant la survenue de ces situations. Dix items servent à mesurer ces deux éléments qui forment la variable appelée «pensées dépressives» liées à la prise en charge (Depressive Cognition Index). Les aidants qui rapportent de telles pensées à un degré élevé éprouvent aussi un degré élevé de dépression, mais seulement si la satisfaction du soutien est peu élevée. Un soutien satisfaisant sert donc de tampon permettant d'atténuer le lien entre les pensées dépressives liées à la prise en charge et la dépression.

En examinant les derniers résultats que nous venons de rapporter, on peut se demander s'il existe certaines contradictions. En effet, nous avons vu que le soutien satisfaisant comme le soutien dérangeant s'avèrent liés au bien-être tandis que le soutien aidant ne l'est pas. Cette observation amène Pagel et al. (1987) à examiner les liens entre la satisfaction, le soutien aidant et dérangeant. ${ }^{2}$ Pour ce faire, ils traitent la satisfaction comme une variable dépendante et ils vérifient dans quelle mesure le soutien aidant et dérangeant déterminent la satisfaction. Les auteurs observent que le soutien dérangeant contribue à la variation de la satisfaction (bêta $=-0,49$ ), ce qui n'est pas le cas pour le soutien aidant (bêta $=0,04$ ). Les auteurs concluent que lorsqu'une personne évalue la satisfaction du soutien, elle tiendrait compte davantage de l'aspect dérangeant du soutien lorsqu'elle se prononce. Cette observation expliquerait pourquoi l'aspect aidant n'est pas lié au bien-être tandis que l'aspect dérangeant l'est.

- En ce qui a trait à la suffisance de soutien, nous observons un plus grand nombre de cas où les liens sont significatif́s plutôt que non significatifs. Il s'avère même que dans l'étude de George et Gwyther (1986), cette situation s'observe dans six des sept aspects mesurés du bien-être (sont inclus les résultats de Clipp et George, $1990 \mathrm{a}$, puisqu'il s'agit du même échantillon). Selon cette étude, les aidants ayant besoin de plus de soutien ont un bien-être plus fragile (George \& Gwyther, 1986) et ils ont tendance à consommer plus de psychotropes (Clipp \& George, 1990a) que ceux rapportant ne pas avoir besoin de plus d'aide. Dans l'étude de Scott et al. (1986), les aidants qui perçoivent le soutien reçu comme suffisant éprouvent moins de fardeau que ceux qui disent ne pas en recevoir suffisamment ou en recevoir plus qu'ils en attendent. Selon les auteurs, ce dernier groupe d'aidants recevraient plus de soutien parce que leur entourage les percevrait particulièrement fragiles.

En somme, les études jettent très peu d'éclairage sur la façon selon laquelle s'opère le lien entre le soutien social et le bien-être. En termes de tendance, en considérant l'ensemble des liens, ces derniers sont plus souvent significatifs lorsque les auteurs utilisent une approche évaluative plutôt que descriptive. En se référant toujours à l'approche descriptive ou évaluative, nous avons examiné chacune des variables dépendantes de bien-être. Nous observons ausși que les liens sont plus souvent significatifs dans le cas d'un approche évaluative. La consommation de psychotropes et l'impact négatif sur la vie familiale constituent les seules variables dépendantes d'exception. Puisque chacune de ces deux variables ne fait l'objet d'étude que dans une recherche, nous ne pouvons tenter d'expliquer ces exceptions. 


\section{TABLEAU 2}

Liens entre les dimensions du soutien et le bien-être selon des mesures descriptives et évaluatives

\begin{tabular}{|c|c|c|c|c|}
\hline Bien-être & Dépression & Santé mentale & Fardeau & Tension \\
\hline \multicolumn{5}{|l|}{$\begin{array}{l}\text { Mesures: } \\
\text { descriptives }\end{array}$} \\
\hline Taille du réseau & $\begin{array}{l}\text { Fiore et al. } \\
\text { (1983), } 5^{*} \\
\text { Fiore et al, (1986), } \\
\text { NS } \\
\text { Kielcolt-Glaser et } \\
\text { al. (1987), NS }\end{array}$ & $\begin{array}{l}\text { Gilhooly (1984), NS, } \\
\text { NS } \\
\text { Gilleard, Belford, } \\
\text { Gilleard, Whittick, } \\
\text { et Gledhill (1984), NS } \\
\text { Fiore et al. (1986) } \\
\text { NS } \\
\text { Niederehe et Scott } \\
\text { (1987), NS }\end{array}$ & $\begin{array}{l}\text { Zarit et al. (1980) } \\
\text { S* }^{*}\end{array}$ & $\begin{array}{l}\text { Niederehe et Scott } \\
\text { (1987), NS }\end{array}$ \\
\hline Confident & & $\begin{array}{l}\text { Niederehe et Scott } \\
\text { (1987), S* } \\
\text { Pruchno (1990), NS }\end{array}$ & Pruchuso (1990), NS & $\begin{array}{l}\text { Niederehe et Scott } \\
\text { (1987), NS }\end{array}$ \\
\hline Aide regue & $\begin{array}{l}\text { Robinson (1989), } \\
\text { NS }\end{array}$ & $\begin{array}{l}\text { Gilhooly (1984), NS, } \\
\text { NS } \\
\text { Gilleard, Belford, } \\
\text { Gilleard, Whittick, } \\
\text { et Gledhill (1984), } \\
\text { NS } \\
\text { Niederehe et Scott } \\
\text { (1987), NS } \\
\text { Pruchno (1990), NS }\end{array}$ & $\begin{array}{l}\text { Gilleard, Gilleard, } \\
\text { Gledhill, et } \\
\text { Whittick (1984b), } \\
\text { NS } \\
\text { Zarit et al. (1986), } \\
\text { NS } \\
\text { Pruchno (1990), NS }\end{array}$ & $\begin{array}{l}\text { Gilleard, Gilleard, } \\
\text { Gledhill, et } \\
\text { Whittick (1984)b, } \\
\text { NS } \\
\text { Niederehe et Seott } \\
\text { (1987), NS }\end{array}$ \\
\hline
\end{tabular}

\begin{tabular}{|c|c|c|}
\hline $\begin{array}{l}\text { Disponibilité } \\
\text { du soutien }\end{array}$ & $\begin{array}{l}\text { Fiore et al, (1986), Fiore et al. (1986), } \\
\text { NS }\end{array}$ & \\
\hline \multicolumn{3}{|l|}{$\begin{array}{c}\text { Mesures } \\
\text { évaluatives }\end{array}$} \\
\hline Soutien aidant & $\begin{array}{l}\text { Fiore et al. (1983), Niederehe et Scott } \\
\text { NS (1987), NS } \\
\text { Kielcolt-Glaser et } \\
\text { al. (1988), NS } \\
\text { Pagel et al. (1987), } \\
\text { NS }\end{array}$ & $\begin{array}{l}\text { Niederehe et Scont } \\
\text { (1987), NS }\end{array}$ \\
\hline $\begin{array}{l}\text { Soutien } \\
\text { dérangeant }\end{array}$ & $\begin{array}{l}\text { Fiore et al, (1983), } \\
\mathrm{S}^{*} \text { pour chacun } \\
\text { des einq types } \\
\text { de soutien } \\
\text { Kielcolt-Glaser et } \\
\text { al. (1988), S* } \\
\text { Pagel et al. (1987), } \\
\text { S* }^{*}\end{array}$ & \\
\hline
\end{tabular}




\section{TABLEAU 2}

Liens entre les dimensions du soutien et le bien-être selon des mesures descriptives et évaluatives

\begin{tabular}{|c|c|c|c|c|c|}
\hline $\begin{array}{l}\text { Satisfaction } \\
\text { de vie }\end{array}$ & $\begin{array}{c}\text { Vie } \\
\text { sociale }\end{array}$ & $\begin{array}{l}\text { Impact négatif } \\
\text { sur vie famillale }\end{array}$ & $\begin{array}{c}\text { Etat } \\
\text { de santé }\end{array}$ & $\begin{array}{c}\text { Consommation } \\
\text { de } \\
\text { psychotropes }\end{array}$ & Autres \\
\hline
\end{tabular}

$\begin{array}{lll}\begin{array}{l}\text { Niederehe et } \\ \text { Scott (1987), } \\ \text { NS }\end{array} & \begin{array}{l}\text { Niederehe et } \\ \text { Scott (1987), } \\ \text { S* }\end{array} & \begin{array}{l}\text { Niederehe et } \\ \text { Scott (1987), S* }\end{array} \\ \begin{array}{lll}\text { Niederehe et } & \text { Niederehe et } & \text { Niederehe et } \\ \text { Scott (1987), } & \text { Scott (1987), } & \text { Scott (1987), } \\ \text { S* } & \text { S* } & \text { NS }\end{array}\end{array}$

$\begin{array}{ll}\text { Clipp et } & \begin{array}{l}\text { Quayhagen et } \\ \text { George } \\ \text { (1990a), S*a }\end{array} \\ & \begin{array}{l}\text { Quayhagen } \\ \text { (1988), S* } \\ \text { Indice composé } \\ \text { de bien-être }\end{array}\end{array}$

$\begin{array}{lll}\text { Niederehe et } & \text { Niederehe et } & \text { Niederehe et } \\ \text { Scott (1987), } & \text { Scott (1987), } & \text { Scott (1987), } \\ \text { NS } & \text { NS } & \text { NS }\end{array}$

Fiore et al.

(1986), NS

$\begin{array}{lll}\text { Niederehe et } & \text { Niederehe et } & \text { Niederehe et } \\ \text { Scott (1987), } & \text { Scott (1987), } & \text { Scott (1987), } \\ \text { NS } & \text { NS } & \text { NS }\end{array}$




\section{TABLEAU 2 (suite)}

Liens entre les dimensions du soutien et le bien-être selon des mesures descriptives et évaluatives

\begin{tabular}{l|lll}
\hline Bien-être & Dépression & Santé mentale & \multicolumn{1}{c}{ Fardeau } \\
\hline Conflit & & $\begin{array}{l}\text { Scott et al. } \\
(1985), \mathrm{s} * \mathrm{~b}\end{array}$ \\
\hline
\end{tabular}

\begin{tabular}{|c|c|c|c|c|}
\hline $\begin{array}{l}\text { Satisfaction/ } \\
\text { insatisfaction }\end{array}$ & $\begin{array}{l}\text { Fiore et al. (1986), } \\
\text { S* }^{*} \\
\text { Pagel et Becker } \\
\text { (1987), S* } \\
\text { Pagel et Becker } \\
\text { (1987), effet } \\
\text { tampon (soutien } \\
\text { sur pensés dé- } \\
\text { pressives), } \text { S* }^{*}\end{array}$ & $\begin{array}{l}\text { Gilhooly (1984), } \\
\text { S*, NS } \\
\text { Fiore et al. (1986), } \\
\text { S* }^{*} \\
\text { Niederehe et Scott } \\
\text { (1987), S* }\end{array}$ & & $\begin{array}{l}\text { Niederefie et Scott } \\
\text { (1987), S* }\end{array}$ \\
\hline Réciprocité & $\begin{array}{l}\text { Fiore et al. } \\
(1986), \mathrm{S}^{*}\end{array}$ & $\begin{array}{l}\text { Fiore et al. } \\
\text { (1986), NS }\end{array}$ & & \\
\hline $\begin{array}{l}\text { Suffisance du } \\
\text { soutien }\end{array}$ & $\begin{array}{l}\text { Robinson (1989), } \\
\text { NS }\end{array}$ & $\begin{array}{l}\text { George et Gwyther } \\
(1986), S^{*}, S^{*}\end{array}$ & $\begin{array}{l}\text { Zarit et al. (1986), } \\
\text { NS } \\
\text { Scott et al. (1986), } \\
\text { S.b }\end{array}$ & \\
\hline $\begin{array}{l}\text { Score composé } \\
\text { du soutien }\end{array}$ & $\begin{array}{l}\text { Haley et al. } \\
\text { (1987), NS } \\
\text { Morris et al. } \\
\text { (1989), } \mathrm{S}^{*}\end{array}$ & & & $\begin{array}{l}\text { Morris et al. } \\
\text { (1989), S* }\end{array}$ \\
\hline
\end{tabular}

Toutefois, bien que les liens significatifs soient plus nombreux dans le cas des mesures évaluatives, une mise en garde s'impose en ce qui a trait au chevauchement conceptuel possible entre les variables évaluatives de soutien et de bien-être, ce qui peut donner lieu à des relations artificielles entre ces deux construits. En effet, plusieurs auteurs soulignent que les mesures évaluatives, par comparaison aux mesures descriptives, sont plus influencées par des traits de personnalité tels que le degré d'estime de soi ou l'humeur dépressive (Barrera, 1986; House, 1981; Qureshi, 1990; Veiel, 1985). Comme nous l'avons vu, la dépression est souvent retenue comme variable de bien-être. De plus, comme nous l'avons mentionné (voir mesure de soutien), dans une étude seulement, les chercheurs vérifient nominalement la possibilité de chevauchement d'items entre les mesures de soutien et de bien-être (Fiore et al., 1986). Quoiqu'il en soit, selon Gottlieb et Selby (1989), ces observations ne doivent pas laisser l'impression que le soutien social constitue une caractéristique de la personne plutôt qu'une ressource qui existe au cours d'échanges interpersonnels. Selon Gottlieb (1985): 


\section{TABLEAU 2 (suite)}

Liens entre les dimensions du soutien et le bien-être selon des mesures descriptives et évaluatives

$\begin{array}{ccccc}\begin{array}{c}\text { Satisfaction } \\ \text { de vie }\end{array} & \text { Vie } & \text { Impact négatif } & \text { Etat } & \text { Consommation } \\ \text { sur vie familiale } & \text { de santé } & \begin{array}{c}\text { de } \\ \text { psychotropes }\end{array} & \text { Autres }\end{array}$

\section{Fiore et al.}

(1986), NS

$\begin{array}{lll}\text { Niederehe et } & \text { Niederehe et } & \text { Niederehe et } \\ \text { Scott, S* } & \text { Scott, S* } & \text { Scott (1987), } \\ & & \text { NS }\end{array}$

\begin{tabular}{|c|c|c|c|}
\hline $\begin{array}{l}\text { George et } \\
\text { Gwyther } \\
(1986), S^{*}\end{array}$ & $\begin{array}{l}\text { George et } \\
\text { Gwyther } \\
(1986), \mathrm{S} \text { " }\end{array}$ & $\begin{array}{l}\text { George et } \\
\text { Gwyther } \\
\text { (1986), } \mathrm{S}^{*} \\
\text { (perception de } \\
\text { sante), NS } \\
\text { (visites au } \\
\text { medecin) }\end{array}$ & $\begin{array}{l}\text { Clipp et } \\
\text { George } \\
\text { (1990a), } 5^{*}\end{array}$ \\
\hline
\end{tabular}

Haley et al.

(1987), S*
Haley et al.

(1987), S* $^{*}$

S* (relation significative et positive); NS (non-significative); a (relation significative et négative); b (moyennes nominatement différentes mais pas de verification statistique).

une telle perspective diminue les possibilités d'apprendre sur la dynamique interpersonnelle des relations intimes ou sur la façon d'activer et d'utiliser le soutien potentiel d'un réseau social en regard du processus de coping (p. 357 , notre traduction).

Examinons maintenant quelques recherches relevées qui traitent de dimensions peu étudiées, mais qu'il importe de considérer selon les écrits sur le soutien social en général. Scott et al. (1985) sont les seuls à examiner les conflits entre les aidants principaux et les membres de la famille, en interrogeant individuellement les deux groupes. Les aidants ne rapportent pas un grand nombre de conflits et ils les discutent rarement avec les membres de la famille, bien que les conflits soient une source importante de stress pour eux. Les aspects conflictuels les plus courants concernent le nombre de visites au parent malade ou à eux-mêmes, la perception de la gravité de la condition du parent malade et les attentes différentes concernant les soins à prodiguer. Les aidants ainsi que les membres de la famille qui ne vivent pas de conflit éprouvent moins de fardeau que ceux qui en 
ont. Lorsque l'aidant et le membre de la famille discutent des conflits, leur fardeau respectif est moins élevé que dans les cas où les conflits ne sont pas discutés. Les auteurs concluent à l'importance de considérer les conflits et de s'enquérir sur la manière dont ils sont envisagés par la famille. D'ailleurs, l'impact de la prise en charge sur la vie familiale demeure un champ très peu exploré.

Une seule recherche porte sur la durée du soutien pendant une période d'un an (Clipp \& George, 1990b). Les auteurs examinent les prédicteurs de la durée du soutien, lequel est considéré selon deux dimensions, soit la perception d'avoir besoin ou non de plus d'aide et la fréquence à laquelle l'aide instrumentale est reçue. Les prédicteurs sont des caractéristiques socio-démographiques (âge, revenu, etc.) et l'ampleur de la situation de besoin des aidants. Pour déterminer l'ampleur de cette situation, nous retrouvons, notamment, des critères de santé mentale (degré de stress, d'affect négatif, consommation de psychotropes, etc.), de santé physique et le fait que le parent malade soit à domicile ou en hébergement. En ce qui a trait à la perception d'avoir besoin de plus d'aide, deux patrons de durée se dessinent. Il y a les aidants qui rapportent ne pas avoir besoin de plus d'aide ( $26 \%$ ) et ceux ( $42 \%$ ) qui en ont besoin de plus, et leur situation ne change pas durant la période d'un an. Selon les prédicteurs retenus et en comparant ces deux groupes, les aidants voulant plus d'aide consomment plus de psychotropes, leur affect s'avêre plus négatif, et ils sont plus nombreux à soigner leur parent malade à domicile. Les aidants qui disent recevoir suffisamment d'aide ont, tout compte fait, une bonne santé mentale et physique, et ils sont plus nombreux à avoir leur parent malade hébergé. Ces observations font dire aux auteurs que les aidants en situation de plus grand besoin sont ceux qui, selon leur perception, ne reçoivent pas suffisamment d'aide.

Quant à la fréquence de l'aide instrumentale reçue, deux scénarios se dégagent. Il y a, d'une part, les aidants $(26 \%)$ qui reçoivent fréquemment de Paide de façon stable ou ceux $(15 \%)$ dont la fréquence augmente. D'autre part, nous retrouvons un groupe d'aidants $(33 \%)$ recevant peu souvent de l'aide durant toute cette période ou ceux $(16 \%)$ dont la fréquence diminue. En comparant ces deux groupes, ceux recevant peu souvent de l'aide ou dont l'aide diminue, sont plus âgés, plus démunis sur le plan financier, plus nombreux à avoir leur parent malade à domicile, et ils éprouvent plus de stress que les autres aidants bénéficiant fréquemment d'aide. Ici aussi, le groupe d'aidants qui se retrouve dans une situation d'un plus grand besoin s'avère celui qui reçoit de l'aide le moins fréquemment. Les auteurs concluent que pour un peu plus des deux tiers des aidants, le soutien demeure stable. De plus, on ne peut présumer que le besoin de soutien déclenche effectivement une mobilisation de l'entourage à en offrir. Il se peut que l'entourage devant leur impuissance à changer la situation, en vienne qu'à s'éloigner des aidants démunis. Il se peut également que d'autres facteurs entrent en jeu quant à cette mobilisation, comme par exemple, la situation où les aidants communiquent peu on non clairement leurs besoins. L'entourage percevrait mal les signes de besoin de soutien ou des demandes peu explicites, ou il ne saurait pas quel soutien offrir ou comment l'assurer. 


\section{CONCLUSION ET RECOMMANDATIONS}

La tendance observée indique que, dans le cas de mesures évaluatives, les liens entre le soutien et le bien-être sont plus souvent significatifs que dans le cas de mesures descriptives. Cependant, la majorité des auteurs vérifient l'hypothèse de la relation entre le soutien social et le bien-être d'une façon trop générale, c'est-à-dire sans suffisamment considérer la diversité des dimensions du soutien ou sans distinguer la spécificité de ces relations en fonction, par exemple, des types de soutien ou des aspects du bien-être. Compte tenu des limites conceptuelles et méthodologiques, les résultats qui se dégagent de ces études doivent être interprétés avec prudence. Mais, il importe surtout de retenir les lacunes sur le plan de la conceptualisation et de la mesure du soutien.

L'augmentation des connaissances peut se réaliser dans les études futures, par une meilleure articulation entre les notions théoriques proposées dans les recherches sur les aidants de personnes atteintes de démence (Lawton, Moss, Kleban, Glicksman, \& Rovine, 1991; Pearlin, Mullan, Semple, \& Skaff, 1990; Pruchno \& Resch, 1989) et celles issues des recherches sur le soutien social en général. De telles études permettraient d'identifier les dimensions critiques à considérer dans l'ètude du soutien auprès de ces aidants. En plus de la nécessité de renforcer les devis des futures études et d'entreprendre des recherches longitudinales, il apparait également essentiel de comprendre comment s'opère l'influence du soutien social.

Dans cette optique, une avenue consisterait à étudier la séquence des relations entre le stress, le soutien, les stratégies de coping, et le bien-être; d'autant plus qu'actuellement certains chercheurs (Haley et al., 1987; Pearlin et al., 1990; Pruchno \& Resch, 1989; Quayhagen \& Quayhagen, 1988) qui étudient la prise en charge de personnes atteintes de démence s'appuient sur un cadre conceptuel du stress. Selon Lazarus et Folkman (1984), un des concepts clés de leur cadre conceptuel est l'évaluation cognitive primaire et secondaire. Face à un événement difficile (stresseur potentiel), une personne évalue la possibilité que cet événement luì soit stressant (évaluation primaire). Dans l'éventualité d'une réponse affirmative, cette personne entreprend une seconde évaluation concernant ses capacités et ses ressources à maîtriser l'événement. Le soutien peut jouer un rôle à deux niveaux différents dans la séquence stress et bien-être (Gottlieb \& Selby, 1989). Le soutien perçu disponible peut intervenir entre l'événement potentiellement stressant et la réaction de stress en faisant en sorte que l'événement soit perçu peu ou moins stressant et, ainsi, atténuer la réaction de stress. Le soutien reçu, s'il est adéquat, peut aussi agir après qu'un événement ait été perçu stressant en favorisant, entre autres, le recours à des stratégies de coping efficaces lesquelles peuvent diminuer le stress éprouvé ou la survenue d'effets adverses liés à la présence de stress. Dans ces séquences, le soutien apparaît comme facteur médiateur (effet tampon). Selon Gottlieb et Selby (1989), les chercheurs sur le soutien social en général reconnaissent que différents stresseurs donnent lieu à différents besoins de soutien et que ueven at different stages of coping with a given stressor, the supportive requirements to be met will differ» (p. 10).

Tel que mentionné dans la première partie de l'article, il conviendrait de distinguer l'échange de soutien lors d'une situation de crise (le plus étudié dans les études sur le soutien en général) de celui ayant cours lors de situations quoti- 
diennes. Selon Veiel (1985), le soutien lors d'une situation de crise servirait à faire face (coping with) à l'impact d'un événement stressant. Son efficacité dépendrait de la correspondance entre le type de crise et le type de soutien. L'échange de soutien lors de situations quotidiennes couvrirait un grand éventail de situations et de types de soutien. Dans le contexte de la prise en charge, les besoins de soutien lors de situations quotidiennes doivent être distingués de ceux générés lors de situations de crise. En effet, nous considérons que la prise en charge de malades déments s'avère une situation de stress chronique au cours de laquelle des situations de crise peuvent survenir. L'accumulation des exigences quotidiennes de la prise en charge constituent les principales sources de stress plutôt que des événements de crise isolés. Nous devrions aussi distinguer le soutien offert au parent malade de celui donné directement à l'aidant principal. Le lien entre le soutien et le bien-être pourrait s'avérer différent.

L'examen des situations faisant en sorte que l'entourage atteint ses limites de soutien, ainsi que celles où il n'est jamais ou peu offert, serait des plus utiles pour guider l'implantation de programmes appropriés aux aidants. Dans cette même visée, nous aurions besoin de connaître les facteurs qui donnent naissance ả l'expression du soutien de la part de l'entourage. Le programme de recherche sur le bien-être des aidants en fonction du soutien s'avère donc chargé.

Il apparaît important que les intervenants adoptent une vue multidimensionnelle du soutien, en examinant à fond les différentes dimensions. Par exemple, l'évaluation de la satisfaction du soutien devrait se faire en considérant différents types de soutien, leur source, et les caractéristiques de la situation où l'échange de soutien a lieu. Une telle démarche permettrait de bien cibler les interventions visant à compléter ou à renforcer les efforts des aidants et ceux de leur famille afin de prévenir leur épuisement.

Nous nous interrogeons sur la terminologie utilisée en ce qui a trait au soutien. En effet, quelle est la pertinence de conserver l'expression soutien "dérangeant»? N'est-ce pas là une juxtaposition de deux mots qui ont un sens opposé? D'ailleurs, on le souligne dans les écrits sur le soutien social. Le terme soutien dans le langage courant signifie aide, appui, protection, et secours. Ne conviendrait-il pas que les chercheurs en viennent qu'à développer un autre construit pour désigner le soutien dérangeant? Dans cette optique, il s'agirait d'identifier les différences entre le soutien et le «soutien dérangeant» (lequel pourrait s'appeler le construit d'échange nuisible ou le construit de s'entre-nuire), et d'examiner comment s'opère l'interaction entre les deux et en regard du bien-être. Au cours de cette démarche, il faudrait s'interroger davantage sur les différentes facettes des échanges «nuisibles». Par exemple, est-ce le contenu de l'échange (types, etc.) qui nuit? Ou encore, est-ce le processus d'échange de soutien, c'est-à-dire la façon selon laquelle cet échange se fait ou s'exprime au cours de l'interaction? En considérant ces réflexions, il deviendrait pertinent d'ajouter un cinquième élément aux propos de House (1981). Selon cet auteur, il faut examiner le «who gives what to whom regarding which problems" (p. 22). Le cinquième élément pourrait être «through which contextual interactionm. Mais au-dessus de ces considérations, nous tenons à souligner que le soutien informel est une ressource à la fois précieuse et fragile et que nous devons assurer des conditions sociales favorables à son expression. 


\section{NOTES}

1. Pour ne pas alourdir la présentation des résultats, nous utilisons l'expression générale «lien significatif» pour désigner les résultats où il $\mathrm{y}$ a une relation significative quel que soit le test statistique utilisẻ (c.g., analyses de corrélation, de régression, tests de $t$, etc.).

2. La mesure de satisfaction (qualité et quantité) du soutien est quelque peu différente de la mesure du soutien aidant et dérangeant. Les auteurs demandent aux aidants de faire la liste des membres de leur réseau social. En considérant chacun des membres énumérés dans leur liste, les aidants doivent se prononcer sur le degré d'aide ou de dérangement en regard de chacun des cinq types de soutien retenus. Dans le cas de la satisfaction, les aidants se prononcent sur le degré de satisfaction vis-à-vis de chacun des cinq types de soutien mais en considérant l'ensemble de leur réseau social (non pas selon chaque membre énuméré dans la liste). D'ailleurs, cette mesure porte le nom de koverall satisfaction with one's social networks.

\section{ABSTRACT}

Many people suffering from dementia live at home, in spite of the demands that such a situation puts on those who care for them. The role of helper is usually assumed by a particular family member who is therefore more likely to feel the impact of the caregiving responsibility. Researchers are concerned with the relationship between the well-being of these helpers and their informal social support. This article presents a critical review of 21 studies of that relationship. The various dimensions of social support considered in the studies are analyzed, as are the methodological limits. A discussion of findings about the relationships between social support and wellbeing completes the analysis. It appears that researchers have a limited conception of social support and assess only a few of its many dimensions in a variety of ways. Very few authors explicitly specify underlying assumptions or a theory relevant to their conceptualization of social support. The weakness and the diversity of this conceptualization are reflected in the measurement tools which consist of a few ad hoc questions on social support in nearly $50 \%$ of the studies. Few studies consider the support availability, duration, conflict, and reciprocity. The buffering effect model of support is examined in one study. Inadequate sample size, unrepresentative samples, and lack of control in regard to confounding variables are among the methodological problems identified. When researchers use an evaluative approach to measure social support, the relationships between support and well-being are more often significant than when a descriptive approach is used. In future studies, researchers should be careful to better articulate the theoretical notions proposed in studies on caring for a family member with dementia at home with those coming from studies on social support in general. It is important for researchers to strengthen their study design and to carry out longitudinal studies; they must also try to understand how the influence of society works. From this perspective, one avenue for the future might involve studying the sequence of relationships between stress, support, coping strategies, and well-being. This is all the more important because at the present time certain studies on the caring of persons with dementia are based on a conceptual framework of stress. Such a framework considers support as a mediating factor. 


\section{REFERENCES}

Barer, B.M., \& Johnson, C.L. (1990). A critique of the caregiving literature. The Gerontologist, 30, 26-29.

Barrera, M. (1981). Social support in the adjustment of pregnant adolescents. Dans B.H. Gottlieb (Ed.), Social networks and social support (pp. 69-96). Beverly Hills, CA: Sage.

Barrera, M. (1986). Distinctions between social support concepts, measures and model5. American Journal of Community Psychology, 14, 413-445.

Barrera, M., Sandler, I., \& Ramsay, T. (1981). Preliminary development of a scale of social support: Studies of college students. American Journal of Community Psychology, 9 , 435-447.

Baumgarten, M. (1989). The health of persons giving care to the demented elderly: A critical review of the literature. Journal of Clinical Epidemiology, 42, 1137-1148.

Beck, A.T., \& Beamsderfer, A. (1974). Assessment of depression: The depression inventory. Modern Problems of Pharmacopsychiatry, 7, 151-170.

Berkman, L.F. (1983). The assessment of social networks and social support in the elderly. Journal of the American Geriatrics Society, 31, 743-749.

Clipp, E.C., \& George, L.K. (1990a). Psychotropic drug use among caregivers of patients with dementia. Journal of American Geriatrics Society, 38, 227-235.

Clipp, E.C., \& George, L.K. (1990b). Caregiver needs and patterns of social support. Journal of Gerontology, 45, S102-111.

Cohen, J. (1977). Statistical power analysis for the behavioral sciences. New York: Academic Press.

Cohen, S., \& Syme, S.L. (1985). Issues in the study and application of social support. Dans S. Cohen \& S.L. Syme (Eds.), Social support and health (pp. 3-22). Orlando: Academic Press.

Cohen, S., \& Wills, T.A. (1985). Stress, social support, and the buffering hypothesis. Psy. chological Bulletin, 98, 310-357.

Derogatis, L.R., Rickels, D., \& Rock, A.F. (1976). The SCL-90 and the MMPI: A step in the validation of a new self-report scale. British Journal of Psychiatry, 128, 280-289.

Dillehay, R.C., \& Sandys, M.R. (1990). Caregivers for Alzheimer's patients: What we are learning from research. International Journal of Aging and Human Development, 30, 263-285.

Duke University Center for the Study of Aging and Human Development. (1978), Multidimensional functional assessment: The OARS methodology, a manual, Durham, NC: Author.

Fiore, J., Becker, J., \& Coppel, D.B. (1983). Social network interactions: A buffer or a stress. American Journal of Community Psychology, 11, 423-439.

Fiore, J., Coppel, D.B., Becker, J, \& Cox, G.B. (1986). Social support as a multifaceted concept: Examination of important dimensions for adjustment. American Journal of Community Psychology, 14, 93-111.

Gallo, J.J. (1990). The effect of social support on depression in caregivers of the elderly. The Journal of Family Practice, 30, 430-440.

George, L.K., \& Gwyther, L.P. (1986). Caregiver well-being: A multidimensional examination of family caregivers of demented adults. The Gerontologist, 26, 253-259.

Gilhooly, M.L.M. (1984). The impact of care-giving on care-givers: Factors associated with the psychological well-being of people supporting a dementing relative in the community. British Journal of Medical Psychology, 37, 35-44.

Gilleard, C.J., Belford, H., Gilleard, E., Whittick, J.E., \& Gledhill, K. (1984). Emotional distress amongst the supporters of the elderly mentally infirm. British Journal of Psychiatry, 145, 172-177.

Gilleard, C.J., Gilleard, E., Gledhill, K., \& Whittick, J. (1984). Caring for the elderly 
mentally infirm at home: A survey of the supporters. Journal of Epidemiology and Community Health, 38, 319-325,

Given, C.W., Collins, C.E., \& Given, B.A. (1988). Sources of stress among families caring for relatives with Alzheimer's disease. Nursing Clinics of North America, 23, 69-82.

Goldberg, D.P. (1978). Manual of the General Health Questionnaire. Windsor: NFERNelson.

Gottlicb, B.H. (1985). Social support and the study of personal relationships. Journal of Social and Personal Relationships, 2, 351-375.

Gottlieb, B.H., \& Selby, P.M. (1989). Social support and mental health: A review of the Virerature (rapport de recherche). Guelph, ON: University of Guelph.

Haley, W.E., Bartolucci, A.A., Levine, E.G., \& Brown, L. (1987). Stress, appraisal, coping and social support as predictors of adaptational outcome among dementia caregivers. Psychology and Aging, 2, 323-330.

Heitzmann, C.A., \& Kaplan, R.M. (1988). Assessment of methods for measuring social support. Health Psychology, 7, 75-109.

Hirsch, B.J. (1980). Natural support systems and coping with major life changes. American Journal of Community Psychology, 8, 159-172.

House, J. (1981). Work stress and social support. Reading, MA: Addison-Wesley.

House, J., \& Kahn, R.L. (1985). Measures and concepts of social support. Dans S. Cohen \& S.L. Syme (Eds.), Social support and health (pp. 83-108). Orlando: Academic Press.

Johnson, C.L., \& Catalano, D.J. (1983). A longitudinal study of family supports to impaired elderly. The Gerontologist, 23, 612-618.

Kielcolt-Glaser, J.K., Dyer, C.S., \& Shuttleworth, E.C. (1988). Upsetting social interactions and distress among Alzheimer's disease family care-givers: A replication and extension. Journal of Community Psychology, 16, 825-837.

Krause, N., Liang, J., \& Yatomi, N. (1989). Satisfaction with social support and depressive symptoms: A panel analysis. Psychology and Aging, 4, 88-97.

Lawton, M.P., Moss, M., Kleban, M.H., Glicksman, A., \& Rovine, M. (1991). A twofactor model of caregiving appraisal and psychological well-being. Journal of Gerontology: Psychological Sciences, 46(4), P181-189.

Lazarus, R.S., \& Folkman, S. (1984). Stress, appraisal, and coping. New York: Springer.

Malonebeach, E.E., \& Zarit, S.H. (1991). Current research issues in caregiving to the elderly. International Journal of Aging and Human Development, 32, 103-114.

Montgomery, R.J.V. (1990). Investigating caregiver burden. Dans K.S. Markides \& C.I. Cooper (Eds.), Aging, stress and health (pp. 201-216). New York: John Wiley \& Sons.

Moos, R.H. Cronkite, R.C., Billings, A.G., \& Finney, J.W. (1984). Health and Daily Living Form Manual. Stanford, CA: Stanford University School of Medicine, Department of Psychiatry and Behavioral Sciences, Social Ecology Laboratory.

Morris, L.W., Morris, R.G., \& Britton, P.G. (1989). Social support networks and formal support as factors influencing the psychological adjustment of spouse caregivers of dementia sufferers. International Journal of Geriatric Psychiatry, 4, 47-51.

Morris, R.G., Morris, L.W., \& Britton, P.G. (1988). Factors affecting the emotional wellbeing of the caregivers of dementia sufferers. British Journal of Psychiatry, 153 , 147-156.

Niederehe, G., \& Scott, J. (1987). Psychological and family factors influencing caregiver stress in senile dementia. The Southwestern, The Journal of Aging for the Southwest, 4, 48-58.

Ory, M.G., Williams, T.F., Emr, M., Lebowitz, B., Rabins, P., Salloway, J., SlussRadbaugh, T., Wolff, E., \& Zarit, S. (1985). Families, informal supports, and Alzheimer's disease. Research on Aging, 7, 623-644.

Pagel, M.D., \& Becker, J. (1987). Depressive thinking and depression: Relations with personality and social resources. Journal of Personality and Social Psychology, 5. 1043-1052. 
Pagel, M.D., Erdly, W.W., \& Becker, J. (1987). Social networks: We get by (and in spite of) a litye help from our friends. Journal of Personality and Social Psychology, 53, 793-804.

Pearlin, L.I., Mullan, J.T., Semple, S.J., \& Skaff, M.M. (1990). Caregiving and the stress process: An overview of concepts and their measures. The Gerontologist, 30, 583-594.

Pruchno, R.A. (1990). The effects of help pattern on the mental health of spouse caregivers. Research on Aging, 12, 57-71.

Pruchno, R.A., \& Resch, N.L. (1989). Mental health of caregiving spouse: Coping as a mediator, moderator or main effect? Psychology and Aging, 4, 454-463.

Quayhagen, M.P., \& Quayhagen, M. (1988). Alzheimer's stress: Coping with the caregiving role. The Gerontologist, 28, 391-396.

Qureshi, H. (1990). Social support. Dans S.M. Peace (Ed.), Researching social gerontology (pp. 32-45), London: Sage.

Robinson, K.M. (1989). Predictors of depression among wife caregivers. Nursing Research, $38,359-363$.

Rook, K.S. (1984). The negative side of social interaction: Impact on psychological wellbeing. Journal of Personality and Sacial Psychology, 46, 1097-1108.

Ryan, M.C., \& Austin, A.G. (1989). Social supports and social networks in the aged. IMAGE: Journal of Nursing Scholarship, 3, 176-180.

Sandler, I.N., \& Barrera, M. (1984). Toward a multimethod approach to assessing the effects of social support. American Journal of Community Psychology, 12, 37-52.

Schaefer, C., Coyne, J.C., \& Lazarus, R. (1981). The health related functions of social support. Journal of Behavioral Medicine, 4, 381-406.

Scott, J., Wiegand, G., \& Niederehe, G. (1984, November). Measuring behavioral prob. lems and dimensions of family caregiving in senile dementia. Paper presented at the 37 th Annual Scientific Meeting of the Gerontological Society of America, San Antonio, TX.

Scott, J.P., Roberto, K.A., Hutton, T., \& Slack, D.M. (1985). Family conflicts in caring for the Alzheimer's patient. Dans J.T. Hutton \& A.D. Kenny (Eds.), Neurology and Neurobiology (pp. 77-86). New York: A, R. Liss.

Scott, J.P., Roberto, K.A., \& Hutton, T. (1986). Families of Alzheimer's victims: Family support to the caregivers. Journal of American Geriatrics Society, 34, 348-354.

Shanas, E. (1979). Social myth as hypothesis: The case of the family relations of old people. The Gerontologist, 19, 3-9.

Shinn, M., Lehmann, S., \& Wong, N.W. (1984). Social interaction and social support. Journal of Social Issues, 40, 55-76.

Stewart, M.J. (1989). Social support instruments created by nurse investigators. Nursing Research, 38, 268-275.

Tardy, C.H. (1985). Social support measurement. American Journal of Community Psychology, 13, 187-202.

Tennstedt, S.L., McKinlay, J.B., \& Sullivan, L.M. (1989). Informal care for frail elders: The role of secondary caregivers. The Gerontological Society of America, 29, 677-691.

Tilden, V.P. (1985). Issues of conceptualization and measurement of social support in the construction of nursing theory. Research in Nursing and Health, 8, 199-206.

Tilden, V.P., \& Galyen, R.D. (1987). Cost and conflict: The darker side of social support. Western Journal of Nursing Research, 9, 9-18.

Tilden, V.P., \& Stewart, B.J. (1985). Technical notes. Problems in measuring reciprocity with difference scores. Western Journal of Nursing Research, 7, 381-385.

Veiel, H.O.F. (1985). Dimensions of social support: A conceptual framework for research. Social Psychiatry, 20, 156-162.

Ward, R.A. (1985). Informal networks and well-being in later life: A research agenda. The Gerontologist, 25, 55-61.

Wentkowski, G.J. (1981), Reciprocity and the coping strategies of older people: Cultural 


\section{REVUE CRITIQUE D'ETUDES SUR LE SOUTIEN SOCIAL}

dimensions of network building. The Gerontologist, 21, 600-609.

Wethington, E., \& Kessler, R.C. (1986). Perceived support, received support, and adjustment to stressful life events. Journal of Health and Social Behavior, 27, 78-89.

Zarit, S.H., Orr, N.K., \& Zarit, J.M. (1985). The hidden victims of Alzheimer's disease, New York: New York University Press.

Zarit, S.H., Reever, K.E., \& Bach-Peterson, J. (1980). Relatives of impaired elderly: Correlates of feelings of burden. The Gerontologist, 20, 649-655.

Zarit, S.H., Tood, P.A., \& Zarit, J.M. (1986). Subjective burden of husbands and wives as caregivers: A longitudinal study. The Gerontologist, 26, 260-266. 